\title{
A Static Analysis of PKI-Based Systems
}

\author{
Benjamin Aziz ${ }^{1}$, David Gray ${ }^{2}$, and Geoff Hamilton ${ }^{2}$ \\ 1 Department of Computing, Imperial College, \\ 180 Queen's Gate, London SW7 2AZ, U.K \\ baziz@doc.ic.ac.uk \\ 2 School of Computing, Dublin City University, Dublin 9, Ireland \\ \{dgray, hamilton\}@computing.dcu.ie
}

\begin{abstract}
This paper presents a non-uniform static analysis for SPIKY, an extension of the spi calculus with capabilities for PKI operations. The analysis, which follows a denotational framework, captures the property of term substitutions resulting from communications, cryptographic and PKI capabilities. The results of the analysis are used to formalise definitions of two security properties: the term secrecy and (un)certified peer-entity participation.
\end{abstract}

\section{Introduction}

In [7], an extension of the spi calculus [1] called the SPIKY language was introduced to clarify some of the issues related to key usage in spi calculus protocol specifications, like the validity of key-user bindings and users' ownership of processes, by making use of special primitives for performing Public-Key Infrastructure (PKI) key-retrieval operations. These included primitives for the retrieval of private and public parts of key pairs belonging to PKI users registered in the domain of the underlying PKI state. The primitives express both certified and uncertified public key-user bindings. Uncertified bindings are necessary for the modelling of protocols designed to run over devices with relatively limited computational power, e.g. handheld PDAs.

In this paper, we construct a non-uniform static analysis for the SPIKY language that captures the property of term substitutions occurring in PKI systems. In particular, the analysis captures PKI users sending and obtaining the substituted terms. Based on this information, it is possible to formalise security properties like for example, whether a user is capable of learning a term, and whether a user can (possibly) confirm that another user has participated in an instance of some protocol. To demonstrate this latter property, consider the following simple protocol between $A$ and $B$, assuming they both know each other's public keys, $K_{A}^{+}$and $K_{B}^{+}$, and $N_{A}, N_{B}$ are fresh nonces:

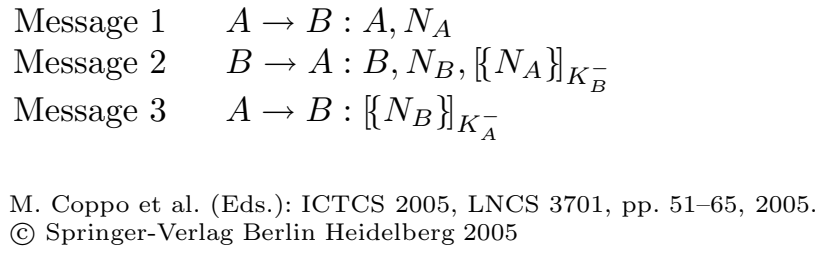


A specification of this protocol in the spi calculus will necessarily make the assumption that the public key $K_{B}^{+}$(respectively $K_{A}^{+}$) must be validly bound to $B$ (respectively $A$ ), and that the partial correctness property of the protocol terminating will ensure that $B$ (respectively $A$ ) participated in the protocol, even in the presence of middle-man attacks. Using the key retrieval capabilities of SPIKY, it is possible to assert this binding formally. Moreover, our static analysis will formally detect the fact that (un)certified public keys were used to arrive at the fact that $B$ (respectively $A$ ) participated in the protocol, and that the private keys used in the signatures were not leaked to third parties.

The static analysis presented in this paper is directly related to previous analyses $[3,4,5,6]$, which were designed to capture the term substitution property for systems specified in the $\pi$-calculus and the spi calculus. The main novelty about the current analysis is that it can deal with systems equipped with key retrieval capabilities over the underlying PKI state.

The rest of the paper is organised as follows. In Section 2 we review the syntax of the SPIKY language. In Section 3 we extend the domain model of [4] to SPIKY processes and we build a denotational semantics for the language. In Section 4, we give an extended semantics that captures the property of term substitutions in SPIKY language. This semantics is safely abstracted in Section 5. In Section 6, we formalise the security properties based on the results of the previous section and in Section 7, we review a simple example of a mobile authentication protocol. Finally, in Section 8, we conclude the paper.

\section{The SPIKY Language}

We review here the syntax of the SPIKY language introduced in [7]. The syntax consists of terms, processes, systems and protocols, as shown in Figure 1. The syntax of terms and processes is mostly similar to that of the spi calculus, except for the following differences, which occur in the syntax of processes:

- The signature with recovery validation process, case $L$ of $[\{x\}\}_{N}$ in $P$, is similar to its counterpart in the spi calculus, whereas the signature with appendix validation process, $\left[[\{L, M\}]_{N}\right] P$, behaves as $P$ only if $L$ is the signature $[\{M\}]_{K^{-}}$and $\mathrm{N}$ is the public key $K^{+}$, otherwise, the process will block.

- Abstraction instantiations, $A(M)$, substitute $x$ in the definition, $A(x) \triangleq P$, where $x$ is bound to $P$, by the term $M$ yielding a process $P[M / x]$. In order to simplify our static analysis, we consider only non-recursive definitions.

- The processes, let $x=\operatorname{private}(M)$ in $P$, let $x=\operatorname{public}(M)$ in $P$ and let $x=\operatorname{certified}(M)$ in $P$, attempt to retrieve the private, uncertified and certified public keys, respectively, of the PKI user, $M$. If successful, the retrieved key, $k$, substitutes $x$, and the process continues as $P[k / x]$, otherwise, the process blocks. The success or failure of these key-retrieval processes depends on the identity of the owner of that process.

In addition to terms and processes, systems formalise the idea of the ownership of a user, $N$, to a process, $P$, written as $\lceil P\rceil^{N}$, where $N$ must reduce to an 


\begin{tabular}{|c|c|c|}
\hline$L, M, N::=$ & $\begin{array}{l}a, b, c, k, m, n \in \mathcal{N} \\
x, y, z, v, w \in \mathcal{V} \\
A, B, C, U \in \mathcal{A} \mathcal{G} \\
\{M\}_{N} \\
\{[M]\}_{N} \\
{[\{M\}]_{N}} \\
(M, N) \\
M^{+} \\
M^{-}\end{array}$ & $\begin{array}{l}\text { terms } \\
\text { names } \\
\text { variables } \\
\text { agents } \\
\text { symmetric encryption } \\
\text { public-key encryption } \\
\text { digital signature } \\
\text { pair } \\
\text { public key component } \\
\text { private key component }\end{array}$ \\
\hline$P, Q, R \quad::=$ & $\begin{array}{l}\bar{M}\langle N\rangle \cdot P \\
M(x) \cdot P \\
P \mid Q \\
(\nu n) P \\
! P \\
{[M \text { is } N] P} \\
\mathbf{0} \\
\text { let }(x, y)=M \text { in } P \\
\text { case } L \text { of }\{x\}_{N} \text { in } P \\
\text { case } L \text { of }\{[x]\}_{N} \text { in } P \\
\text { case } L \text { of }[\{x\}]_{N} \text { in } P \\
{\left[[\{L, M\}]_{N}\right] P} \\
A(M) \\
\text { let } x=\operatorname{private}(M) \text { in } P \\
\text { let } x=\text { public }(M) \text { in } P \\
\text { let } x=\text { certified }(M) \text { in } P\end{array}$ & $\begin{array}{l}\text { processes } \\
\text { output } \\
\text { input } \\
\text { parallel composition } \\
\text { restriction } \\
\text { replication } \\
\text { match } \\
\text { null } \\
\text { pair splitting } \\
\text { symmetric decryption } \\
\text { public-key decryption } \\
\text { signature with recovery validation } \\
\text { signature with appendix validation } \\
\text { abstraction instantiation } \\
\text { private key retrieval } \\
\text { public key retrieval } \\
\text { certified public key retrieval }\end{array}$ \\
\hline$E, F, G \quad::=$ & $\begin{array}{l}E \mid F \\
(\nu n) E \\
\lceil P\rceil^{N}\end{array}$ & $\begin{array}{l}\text { systems } \\
\text { parallel composition } \\
\text { restriction } \\
\text { process ownership }\end{array}$ \\
\hline Prot & $(\theta, E)$ & $\begin{array}{l}\text { protocols } \\
\text { (PKI state, system) pair }\end{array}$ \\
\hline
\end{tabular}

Fig. 1. The syntax of the SPIKY language

agent's name. Systems can also be composed in parallel and new names can be introduced within the scope of a system. Finally, a protocol is a pair consisting of a PKI state, $\theta: \mathcal{A G} \rightarrow \mathcal{N}$, and a system, E. A PKI state maps distinct names of PKI users (agents) to their corresponding key pairs. The details of the actual binding of users to key pairs are abstracted, as well as the mechanisms for revocation. In general, $\theta$ is assumed to be up to date when a protocol is run.

In the following sections, we assume that the notions of $\alpha$-conversion and term substitution as well as free and bound names and variables of terms and processes all apply as usual. We also assume that there are no occurrences of homonymous bound names and variables. Finally, in any protocol, we assume that there are only finitely many agents, possibly with replicated process behaviour.

\section{A Domain-Theoretic Model}

We introduce here a domain-theoretic model for closed processes in the SPIKY language that is an extension of the model originally given in [4] for the spi calculus. Our model is based on the following predomain equations: 


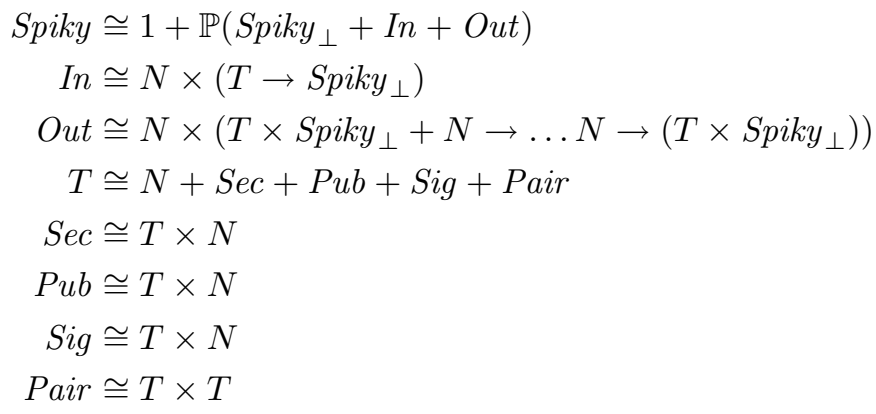

Where $S p i k y_{\perp}$ is the domain of processes, In and Out are the predomains of input and output actions, respectively. Input actions are modelled as pairs; a name, $N$ (the channel), and a function, $T \rightarrow S p i k y_{\perp}$, that can be instantiated with a term, $T$, yielding a process in $S p i k y_{\perp}$. Output actions are divided into free and bound output actions. These are pairs consisting of the channel, $N$, and either another pair, $T \times S p i k y_{\perp}$, denoting the message, $T$, and the residue $S p i k y_{\perp}$ (free outputs), or composed functions, $N \rightarrow \ldots N \rightarrow\left(T \times S p i k y_{\perp}\right)$, that introduce new names to the message, $T$, and the residue, $S p i k y_{\perp}$ (bound outputs). $\mathbb{P}(-)$ is Plotkin's powerdomain [8] applied to the disjoint union of input, output and silent actions (the latter represented by $S p i k y_{\perp}$ ) to construct Spiky. The one-element predomain, 1, representing terminated (deadlocked) processes is adjoined as in [2, Def. 3.4]. The flat predomain of closed terms, $T$, is defined as the disjoint union of the predomains of names, $N$, secret-key ciphers, Sec, public-key ciphers, Pub, digital signatures, Sig, and pairs, Pair. The predomains Sec, Pub and Sig are represented as pairs, $T \times N$, where the term, $T$, is encrypted/signed using the key, $N$. There is no predomain of variables as part of the definition of $T$ since we only deal with closed semantic terms.

The following functions are also defined, leading into Spiky $y_{\perp}[2$, Def. 3.3]:

$$
\begin{aligned}
& \emptyset: 1 \rightarrow \text { Spiky }_{\perp} \\
& \{-\}:\left(\text { Spiky }_{\perp}+\text { In }+ \text { Out }\right)_{\perp} \rightarrow \text { Spiky }_{\perp} \\
& \uplus:\left(S p i k y_{\perp} \times S p i k y_{\perp}\right) \rightarrow S p i k y_{\perp} \\
& \text { new }:\left(N \rightarrow S p i k y_{\perp}\right) \rightarrow S p i k y_{\perp}
\end{aligned}
$$

The empty set, $\emptyset$, is required to represent inactive processes. The singleton map, $\{|-|\}$, creates elements of Spiky $y_{\perp}$ from elements of input, output and silent actions, and $\uplus$, is the standard multiset union operator representing nondeterminism. Finally, new is used to interpret the effects of restriction.

Concrete elements of $t \in T$ include names, $a, b, c$, secret-key ciphers, $\sec (t, k)$, public-key ciphers, $\operatorname{pub}(t, k)$, digital signatures, $\operatorname{sig}(t, k)$, and pairs, $\left(t, t^{\prime}\right)$. Elements $p \in S p i k y_{\perp}$ include the bottom element, $\{\mid \perp\}$, the empty set, $\emptyset$ (where $\{\mid \perp\} \sqsubseteq \emptyset)$, input actions, $\{$ in $(a, \lambda y \cdot p) \mid\}$, free output actions, $\{\mid$ out $(a, t, p)\}$, bound 


\begin{tabular}{|c|c|}
\hline 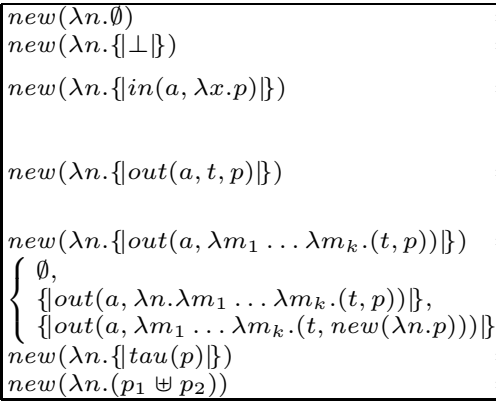 & 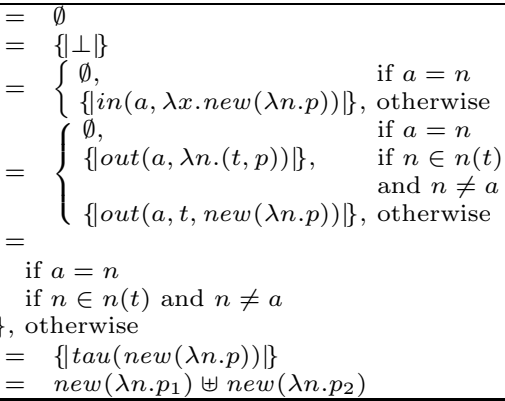 \\
\hline
\end{tabular}

Fig. 2. The concrete definition of new over elements $p \in S p i k y_{\perp}$

output actions, $\left\{\mid \operatorname{out}\left(a, \lambda n_{1} \ldots \lambda n_{m} \cdot(t, p)\right)\right\}$ silent actions, $\{\mid \operatorname{tau}(p)\}$ and sums, $p \uplus q$. The effects of restriction are interpreted by defining new as in Figure 2 . These effects lead to the blocking of processes attempting to communicate over fresh, non-extruded channels and the transformation of free outputs to bound outputs whenever the message of communication is a fresh name. Otherwise, new has no effect and it is simply distributed over $\uplus$.

The denotational semantics for the SPIKY language is given as a semantic function, $\mathcal{S}([E]) \rho \phi_{\mathcal{S}} \theta \in S p i k y_{\perp}$, defined by the set of rules of Figure 3 . The $\theta$ environment is defined as the PKI state of some protocol, such that $\theta(U)$ is a name representing the key pair associated with the user, $U$, where $\theta(U)^{+}$is the public part of that key pair and $\theta(U)^{-}$is the private part. The multiset, $\rho$, is used to hold systems composed in parallel with the analysed system and $\{|-|\}$ and $\uplus$ are overloaded from their definitions in $(10),(11)$ to deal with $\rho$. Furthermore, rule $(\mathcal{R} 0)$ is used to interpret the contents of $\rho$. The environment, $\phi_{\mathcal{S}}: V \rightarrow T$, where $V$ is the flat predomain of variables, captures any term substitutions that occur in the semantics ${ }^{1}$. The special function, $\varphi_{\mathcal{S}}$, returns the semantic value of a term:

$$
\forall \phi_{\mathcal{S}}, M: \varphi_{\mathcal{S}}\left(\phi_{\mathcal{S}}, M\right)= \begin{cases}\phi_{\mathcal{S}}(M), & \text { if } M \in N \wedge M \in V \\ \sec \left(\varphi_{\mathcal{S}}\left(\phi_{\mathcal{S}}, M^{\prime}\right), \varphi_{\mathcal{S}}\left(\phi_{\mathcal{S}}, N\right)\right), & \text { if } M=\left\{M^{\prime}\right\}_{N} \\ \operatorname{pub}\left(\varphi_{\mathcal{S}}\left(\phi_{\mathcal{S}}, M^{\prime}\right), \varphi_{\mathcal{S}}\left(\phi_{\mathcal{S}}, N\right)\right), & \text { if } M=\left\{\left[M^{\prime}\right]\right\}_{N} \\ \operatorname{sig}\left(\varphi_{\mathcal{S}}\left(\phi_{\mathcal{S}}, M^{\prime}\right), \varphi_{\mathcal{S}}\left(\phi_{\mathcal{S}}, N\right)\right), & \text { if } M=\left[\left\{M^{\prime}\right\}\right]_{N} \\ \left(\varphi_{\mathcal{S}}\left(\phi_{\mathcal{S}}, N\right), \varphi_{\mathcal{S}}\left(\phi_{\mathcal{S}}, L\right)\right), & \text { if } M=(N, L)\end{cases}
$$

Rules $(\mathcal{S} 0 A)$ and $(\mathcal{S} 0 B)$ interpret parallelism and restriction between two systems by joining the parallel systems to $\rho$ and using the new operator, respectively. Rules $(\mathcal{S} 1)-(\mathcal{S} 16)$ deal with process ownership by cases. Rule $(\mathcal{S} 1)$, deals with output actions taking into consideration any communications that may occur between the output channel and appropriate input channels guarding processes in $\rho$. The $\phi_{\mathcal{S}}$ is updated appropriately with the substituted semantic elements. Rules $(\mathcal{S} 2)$ deals with input functions leaving out communications since these are considered in $(\mathcal{S} 1)$. Rule $(\mathcal{S} 3)$ interprets directly parallel

\footnotetext{
${ }^{1}$ Note that initially, $\forall u \in V+N: \phi_{\mathcal{S} 0}(u)=u$.
} 


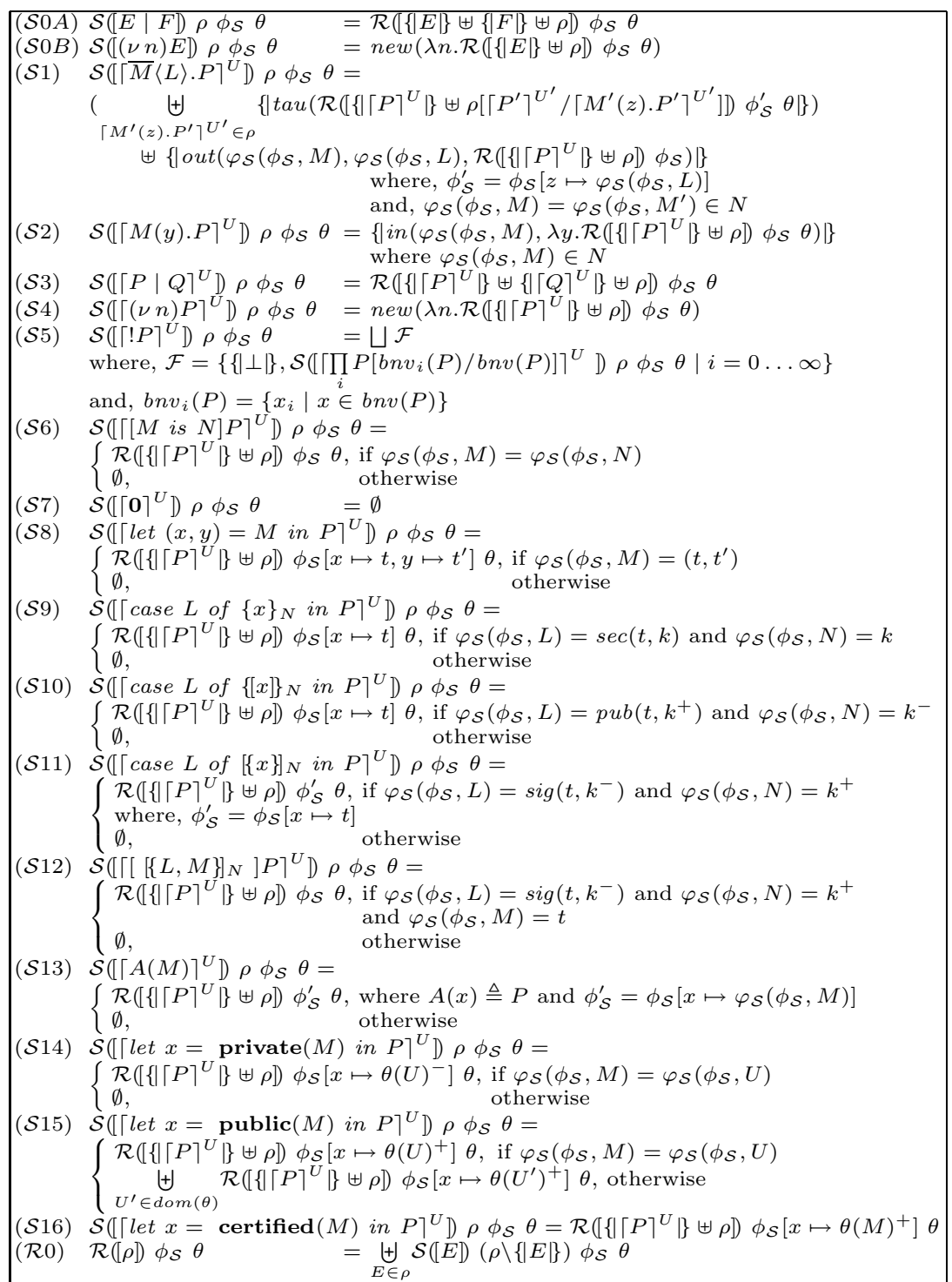

Fig. 3. The standard denotational semantics of the SPIKY language

composition by the addition of the parallel subprocesses to $\rho$. Rule $(\mathcal{S} 4)$ uses new to interpret the meaning of a restriction. Rule $(\mathcal{S} 5)$ interprets a replication, $\lceil! P\rceil^{U}$, as the least upper bound of the infinite poset $\mathcal{F}$. This least upper bound represents the least fixed point meaning of $! P$. Due to the fact that the semantic domain, $S p i k y_{\perp}$, is infinite, the calculation of this least fixed point may not terminate within finite limits. The rule also uses a labelling mechanism to rename all the bound variables and names, bnv $(P)$, of the spawned processes 
by subscripting those variables and names with a number signifying process copy.

Rule $(\mathcal{S} 6)$ resolves the meaning of two terms using $\varphi_{\mathcal{S}}$. Rule $(\mathcal{S} 7)$ interprets the meaning of a null system as the empty set mapping, $\emptyset$. Rule $(\mathcal{S} 8)$ splits the elements of a pair term. Rules $(\mathcal{S} 9)-(\mathcal{S} 12)$ deal with cryptographic systems for the decryption of symmetric and public-key ciphertexts and signatures with recovery and appendix validations. A residual system, $\lceil P\rceil^{U}$, signifying the success of the operation is added to $\rho$, else, if the operation fails, $\emptyset$ is returned instead. Rule $(\mathcal{S} 13)$ interprets the meaning of abstraction instantiations directly by adding the definition to $\rho$ and updating $\phi_{\mathcal{S}}$ with the substituted term. Rules $(\mathcal{S} 14)-(\mathcal{S} 16)$ deal with PKI operations for retrieving private, uncertified and certified public keys. This is done using the PKI state, $\theta$, and the user owning the system, $U$. The uncertified public key operation offers less guarantees (if the owner of the process requires other users' keys), therefore, it may return the public key of any PKI user, $U^{\prime}$, in $\operatorname{dom}(\theta)$. On the other hand, the certified version is always guaranteed to return a valid public key, regardless of the owner's identity.

\section{Non-standard Semantics}

We extend here the standard semantics of the previous section to a non-standard semantics that captures the property of term substitutions. Hence, the meaning of a system is now given as a special environment, $\phi_{\mathcal{E}}: V \rightarrow \wp(T \times A G \times$ $A G)$, which maps each variable of a closed system to a set of triples representing semantic terms that may substitute the variable, and names of PKI users that instantiate and own that variable. Note that $A G$ here represents the flat predomain of agent names corresponding to the set $\mathcal{A G}$. Since the nonstandard semantics is precise (copies of bound names and variables are always distinct), each variable will be mapped to a singleton set per choice of control flow.

A non-standard semantic domain, $D_{\perp}=V \rightarrow \wp(T \times A G \times A G)$, can be constructed, ordered by subset inclusion as follows:

$$
\forall \phi_{\mathcal{E} 1}, \phi_{\mathcal{E} 2} \in D_{\perp}: \phi_{\mathcal{E} 1} \sqsubseteq_{D_{\perp}} \phi_{\mathcal{E} 2} \Leftrightarrow \forall x \in V: \phi_{\mathcal{E} 1}(x) \subseteq \phi_{\mathcal{E} 2}(x)
$$

with the bottom element, $\perp_{D_{\perp}}$, being the null environment, $\phi_{\mathcal{E} 0}$, that maps each variable to the empty set. The union of environments operation, $\cup_{\phi}$, can also be defined as follows:

$$
\forall \phi_{\mathcal{E} 1}, \phi_{\mathcal{E} 2} \in D_{\perp}, x \in V:\left(\phi_{\mathcal{E} 1} \cup_{\phi} \phi_{\mathcal{E} 2}\right)(x)=\phi_{\mathcal{E} 1}(x) \cup \phi_{\mathcal{E} 2}(x)
$$

The non-standard semantics of the SPIKY language is defined using the semantic function, $\mathcal{E}([E]) \rho \phi_{\mathcal{E}} \theta \in D_{\perp}$, as illustrated in Figure 4. The definitions of $\rho$ and $\theta$ are as in Section 3. The definition of the special function, $\varphi_{\mathcal{E}}$ allows for the meaning of a closed term to be computed under some $\phi_{\mathcal{E}}$ : 


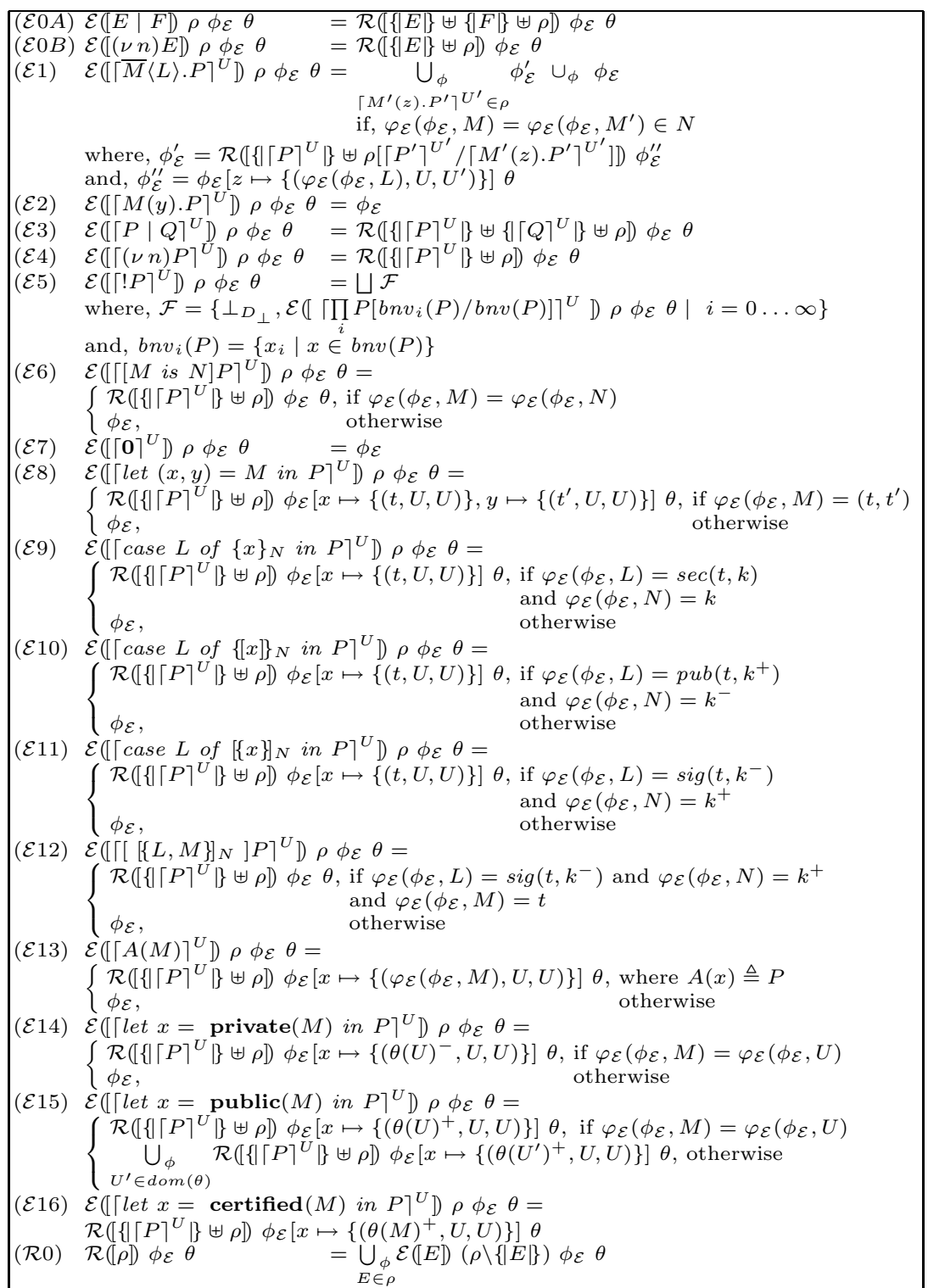

Fig. 4. The non-standard semantics of the SPIKY language

$\varphi_{\mathcal{E}}\left(\phi_{\mathcal{E}}, M\right)^{2}=\left\{\begin{array}{lr}t, & \text { if } M \in V \wedge \\ & \phi_{\mathcal{E}}(M)=\left\{\left(t, U, U^{\prime}\right)\right\} \\ M, & \text { if } M \in N \\ \sec \left(\varphi_{\mathcal{E}}\left(\phi_{\mathcal{E}}, M^{\prime}\right), \varphi_{\mathcal{E}}\left(\phi_{\mathcal{E}}, N\right)\right), & \text { if } M=\left\{M^{\prime}\right\}_{N} \\ \operatorname{pub}\left(\varphi_{\mathcal{E}}\left(\phi_{\mathcal{E}}, M^{\prime}\right), \varphi_{\mathcal{E}}\left(\phi_{\mathcal{E}}, N\right)\right), & \text { if } M=\left\{\left[M^{\prime}\right]\right\}_{N} \\ \operatorname{sig}\left(\varphi_{\mathcal{E}}\left(\phi_{\mathcal{E}}, M^{\prime}\right), \varphi_{\mathcal{E}}\left(\phi_{\mathcal{E}}, N\right)\right), & \text { if } M=\left[\left\{M^{\prime}\right\}\right]_{N} \\ \left(\varphi_{\mathcal{E}}\left(\phi_{\mathcal{E}}, M^{\prime}\right), \varphi_{\mathcal{E}}\left(\phi_{\mathcal{E}}, M^{\prime \prime}\right)\right), & \text { if } M=\left(M^{\prime}, M^{\prime \prime}\right)\end{array}\right.$

${ }^{2}$ Note that the case where $M \in V \wedge \phi_{\mathcal{E}}(M)=\{\}$ will never occur for closed terms. 
The main difference in this semantics as compared to the standard semantics of the previous section is the fact that the meaning of a process is a $\phi_{\mathcal{E}}$ environment rather than an element of $S p i k y_{\perp}$. Note again the difference in performing uncertified versus certified public key retrieval in rules $(\mathcal{E} 15)$ and $(\mathcal{E} 16)$, respectively. In the former case, the owner of a process may obtain any public key stored in $\theta$ when asking for some other user's public key without any guarantees as to the validity of the key-user binding (unless the owner asks for its own public key). In the latter case, this requirement is always guaranteed to return a public key that is validly bound to its user.

\section{Abstract Semantics}

One problem with the non-standard semantics of the previous section is that it is not guaranteed to terminate due to the possibility of infinite behaviour resulting from the presence of replication. Therefore, it is necessary to introduce a safe abstraction that limits the size of the semantic domain. This abstraction is a variation of the abstraction used in $[3,4,6]$.

We begin first by assuming a predomain of tags, Tag, ranged over by $t, \dot{t}, \ddot{t}$, where $t$ is the tag of a generic term, $\dot{t}$ is the tag of a name or a variable, and $\ddot{t}$ is the tag of a complex term (ciphertext, signature, pair). Next, we appropriately $\operatorname{tag} M, M^{\prime}$ in $\bar{N}\langle M\rangle . P$, let $(x, y)=\left(M, M^{\prime}\right)$ in $P$, case $\{M\}_{N}$ of $\{x\}_{N^{\prime}}$ in $P$, case $\{[M]\}_{N}$ of $\{[x]\}_{N^{\prime}}$ in $P$, case $[\{M\}\}_{N}$ of $[\{x\}]_{N^{\prime}}$ in $P, A(M)$, and tag each of private $(M)$, public $(M)$ and certified $(M)$ in the syntax. Furthermore, the following functions are defined over tags and systems:

- value_of $\left(\left\{t_{1}, \ldots, t_{n}\right\}\right)=\left\{M_{1}, \ldots, M_{n}\right\}$, which when applied to a set of tags, $\left\{t_{1}, \ldots, t_{n}\right\}$, returns the corresponding set of terms, $\left\{M_{1}, \ldots, M_{n}\right\}$.

- tags_of $(E)=\left\{t_{1}, \ldots, t_{n}\right\}$, which when applied to a system, $E$, returns its set of tags, $\left\{t_{1}, \ldots, t_{n}\right\}$.

We now introduce the $\alpha_{k, k^{\prime}}$ abstraction function, which keeps to a finite level, the number of copies of bound variables, bound names and tags.

Definition 1. Define $\alpha_{k, k^{\prime}}: \mathbb{N} \times \mathbb{N} \times(V+N+\operatorname{Tag}) \rightarrow\left(V^{\sharp}+N^{\sharp}+T a g^{\sharp}\right)$ :

$\forall M \in(V+N+$ Tag $), i, k, k^{\prime} \in \mathbb{N}: \alpha_{k, k^{\prime}}(M)=\left\{\begin{array}{l}\dot{t}_{k}, \text { if } M=\dot{t}_{i} \in \text { Tag and } i>k \\ \ddot{t}_{k^{\prime}}, \text { if } M=\ddot{t}_{i} \in \text { Tag and } i>k^{\prime} \\ x_{k}, \text { if } M=x_{i} \in V \text { and } i>k \\ a_{k}, \text { if } M=a_{i} \in N \text { and } i>k \\ M, \text { otherwise }\end{array}\right.$

The resulting abstract predomains, $V^{\sharp}, N^{\sharp}$ and Tag $g^{\sharp}$, can be defined as $V^{\sharp}=$ $V \backslash\left\{x_{j} \mid j>k\right\}, N^{\sharp}=N \backslash\left\{a_{j} \mid j>k\right\}$ and Tag $g^{\sharp}=\operatorname{Tag} \backslash\left(\left\{\dot{t}_{j} \mid j>k\right\} \cup\left\{\ddot{t}_{i} \mid\right.\right.$ $\left.i>k^{\prime}\right\}$ ). Informally, $k$ constrains the number of bound variables and names, and tags of primitive terms, whereas $k^{\prime}$ constrains the number of tags of complex terms. In effect, constraining the tags of primitive terms implies limiting the copies of bound names and variables carrying the tags, whereas constraining the number of tags of complex terms means limiting the depth of data structures. 
For example, in the process ! $(\nu n) \bar{a}\left\langle n^{\dot{t}}\right\rangle \mid ! a(x)$, it is possible to spawn infinite

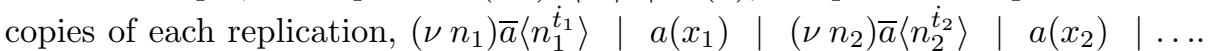
It is clear that $\dot{t}$ is an indicator to the number of copies $n$ has after spawning each process. On the other hand, the process $! a(x) \cdot \bar{a}\left\langle\{x\}_{k}^{\ddot{t}}\right\rangle \mid \bar{a}\langle b\rangle$, which also spawns $a\left(x_{1}\right) \cdot \bar{a}\left\langle\left\{x_{1}\right\}_{k}^{\ddot{t}_{1}}\right\rangle\left|a\left(x_{2}\right) \cdot \bar{a}\left\langle\left\{x_{2}\right\}_{k}^{\ddot{t}_{2}}\right\rangle\right| \bar{a}\langle b\rangle \mid \ldots$ demonstrates the role of $\ddot{t}$ as an indicator to the number of times the ciphertext, $\{x\}_{k}$, is applied to $b$.

Using $\alpha_{k, k^{\prime}}$, we construct $\phi_{\mathcal{A}}: V^{\sharp} \rightarrow \wp\left(T a g^{\sharp} \times A G \times A G\right)$, with a meaning similar to $\phi_{\mathcal{E}}$ in the previous section. Furthermore, a domain, $D_{\perp}^{\sharp}=V^{\sharp} \rightarrow$ $\wp\left(T a g^{\sharp} \times A G \times A G\right)$ is formed as follows:

$$
\forall \phi_{\mathcal{A} 1}, \phi_{\mathcal{A} 2} \in D_{\perp}^{\sharp}, x \in V^{\sharp}: \phi_{\mathcal{A} 1} \sqsubseteq_{D_{\perp}^{\sharp}} \phi_{\mathcal{A} 2} \Leftrightarrow \phi_{\mathcal{A} 1}(x) \subseteq \phi_{\mathcal{A} 2}(x)
$$

with a bottom element, $\perp_{D_{\perp}^{\sharp}}$, representing the null environment, $\phi_{\mathcal{A} 0}$. Taking $D_{\perp}^{\sharp}$ as the abstract semantic domain, we can define the abstract semantics of the SPIKY language using the function, $\mathcal{A}([E]) \rho \phi_{\mathcal{A}} \theta \in D_{\perp}^{\sharp}$, as shown in Figure 5 . The definitions of $\rho$ and $\theta$ are as in the previous sections. The special function, $\varphi_{\mathcal{A}}$, returns a set of terms corresponding to a term, $M$, given substitutions captured by $\phi_{\mathcal{A}}$, as follows:

$\varphi_{\mathcal{A}}\left(\phi_{\mathcal{A}}, M\right)=\varphi_{\mathcal{A}}^{\prime}\left(\phi_{\mathcal{A}}, M^{\prime}\right)_{\{\}}$, where, $M^{\prime}=M\left[\alpha_{k, k^{\prime}}(t) / t\right]\left[\alpha_{k, k^{\prime}}(x) / x\right]\left[\alpha_{k, k^{\prime}}(n) / n\right]$, for all tags, $t$, names, $n$, and variables, $x$, of $M$,

and $\varphi_{\mathcal{A}}^{\prime}\left(\phi_{\mathcal{A}}, M\right)_{s}=$ if $M \in s$ then \{\} else

$$
\begin{cases}\bigcup_{L \in \text { value_of }\left(f s t\left(\phi_{\mathcal{A}}(M)\right)\right)} \varphi_{\mathcal{A}}^{\prime}\left(\phi_{\mathcal{A}}, L\right)_{s \cup\{M\}} & \text { if } M \in \mathcal{V} \\ \{M\}, & \text { if, } M \in \mathcal{N} \\ \left\{\left\{N^{\prime}\right\}_{L^{\prime}}^{t} \mid N^{\prime} \in \varphi_{\mathcal{A}}^{\prime}\left(\phi_{\mathcal{A}}, N\right)_{s \cup\{M\}}, L^{\prime} \in \varphi_{\mathcal{A}}^{\prime}\left(\phi_{\mathcal{A}}, L\right)_{s \cup\{M\}}\right\}, & \text { if, } M=\{N\}_{L}^{t} \\ \left\{\left\{\left[N^{\prime}\right]\right\}_{L^{\prime}}^{t} \mid N^{\prime} \in \varphi_{\mathcal{A}}^{\prime}\left(\phi_{\mathcal{A}}, N\right)_{s \cup\{M\}}, L^{\prime} \in \varphi_{\mathcal{A}}^{\prime}\left(\phi_{\mathcal{A}}, L\right)_{s \cup\{M\}}\right\}, & \text { if, } M=\{[N]\}_{L}^{t} \\ \left\{\left[\left\{N^{\prime}\right\}\right]_{L^{\prime}}^{t} \mid N^{\prime} \in \varphi_{\mathcal{A}}^{\prime}\left(\phi_{\mathcal{A}}, N\right)_{s \cup\{M\}}, L^{\prime} \in \varphi_{\mathcal{A}}^{\prime}\left(\phi_{\mathcal{A}}, L\right)_{s \cup\{M\}}\right\}, & \text { if, } M=[\{N\}]_{L}^{t} \\ \left\{\left(L_{1}^{\prime}, L_{2}^{\prime}\right)^{t} \mid L_{1}^{\prime} \in \varphi_{\mathcal{A}}^{\prime}\left(\phi_{\mathcal{A}}, L_{1}\right)_{s \cup\{M\}}, L_{2}^{\prime} \in \varphi_{\mathcal{A}}^{\prime}\left(\phi_{\mathcal{A}}, L_{2}\right)_{s \cup\{M\}}\right\}, & \end{cases}
$$

We describe a few rules here. Rule $(\mathcal{A} 1)$ deals with the case of output actions, dealing with possible communications with appropriate input actions in $\rho$. The tag of the output message is registered in $\phi_{\mathcal{A}}$ as a value for the input variable. The semantics is imprecise, since $\phi_{\mathcal{A}}$ only captures an abstract tag as a value for an abstract variable. Rule $(\mathcal{A} 5)$ introduces the functions:

ren $(x, i)=$ fold $_{\text {sub }}\left(\right.$ fold $_{\text {sub }} x$ bnv $\left.(x)\right)$ tags_of $(x)$

fold $f$ e $\left\{x_{1}, \ldots, x_{n}\right\}=f\left(x_{n}, \ldots, f\left(x_{1}, e\right) \ldots\right)$

$\operatorname{sub}_{i}$ x $y=y\left[x_{i} / x\right]$

which are used in the definition of the least fixed point meaning of a replicated process. This meaning is defined as the least upper bound of the set $\mathcal{F}$, which 


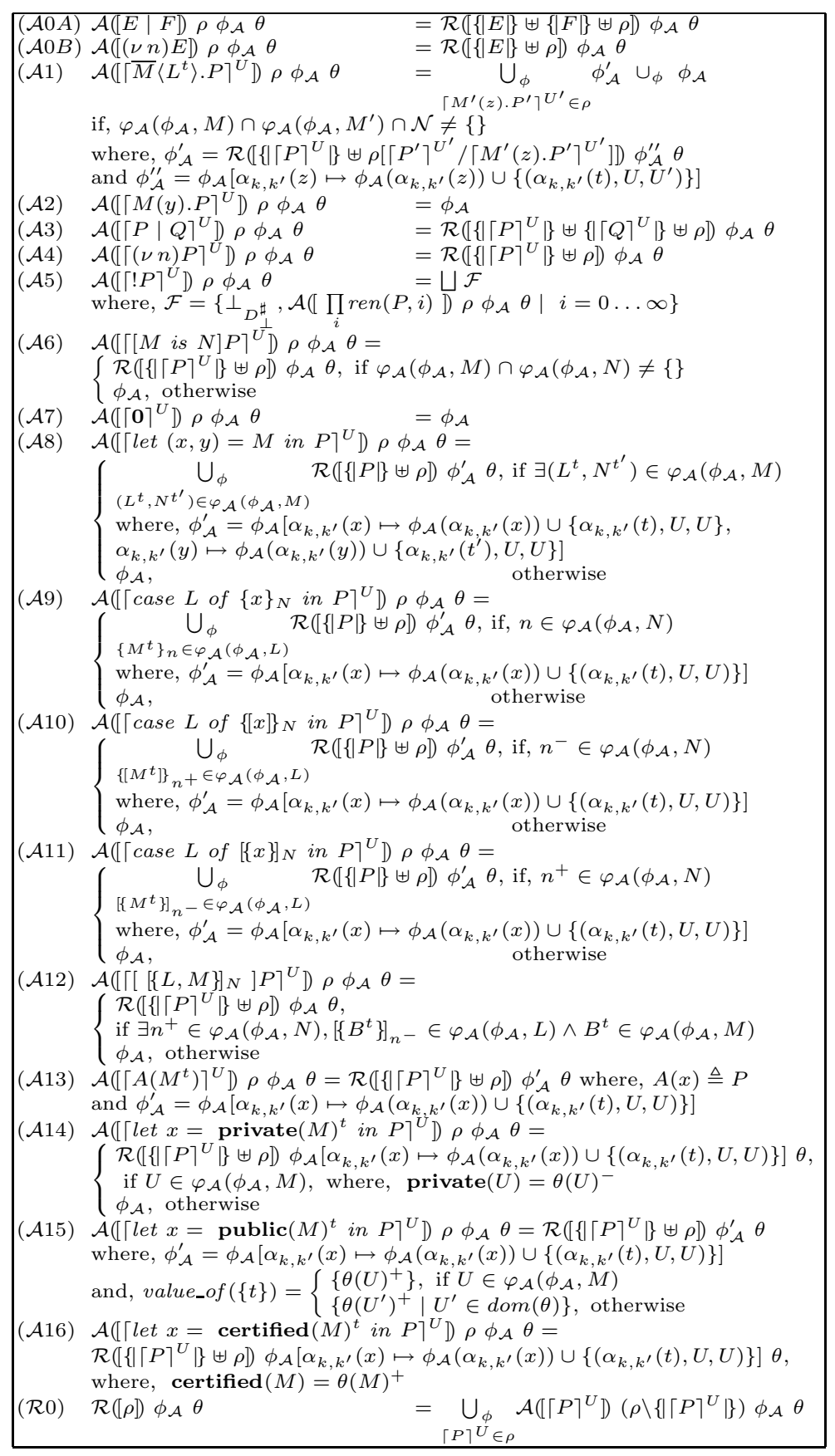

Fig. 5. The abstract semantics of the SPIKY language

can only be finite in this semantic. As a result, the termination of the least fixed point is formalised as follows. 


\section{Theorem 1 (Termination of the least fixed point calculation) The calculation of rule $(\mathcal{A} 5)$ terminates.}

Proof Sketch. To prove the termination property, it is necessary to satisfy two requirements. First, the semantic domain must be finite. This is satisfied by the definition of $D^{\sharp}$, where $T a g^{\sharp}$ and $A G$ are both finite. The second requirement is that $\mathcal{A}\left(\llbracket \prod_{i} P\right\rceil^{U} \rrbracket \rho \phi_{\mathcal{A}} \theta$ must be monotonic over $P$, i.e. $\mathcal{A}\left(\llbracket\left\lceil\prod_{i} P\right\rceil^{U} \rrbracket \rho \phi_{\mathcal{A}} \theta \sqsubseteq_{D_{\perp}^{\sharp}}\right.$ $\left.\left.\mathcal{A}\left(\llbracket \prod_{i+1} P\right\rceil^{U}\right]\right) \rho \phi_{\mathcal{A}} \theta$.

We can state the safety of the abstract semantics by the following theorem.

\section{Theorem 2 (Safety of the abstract semantics)}

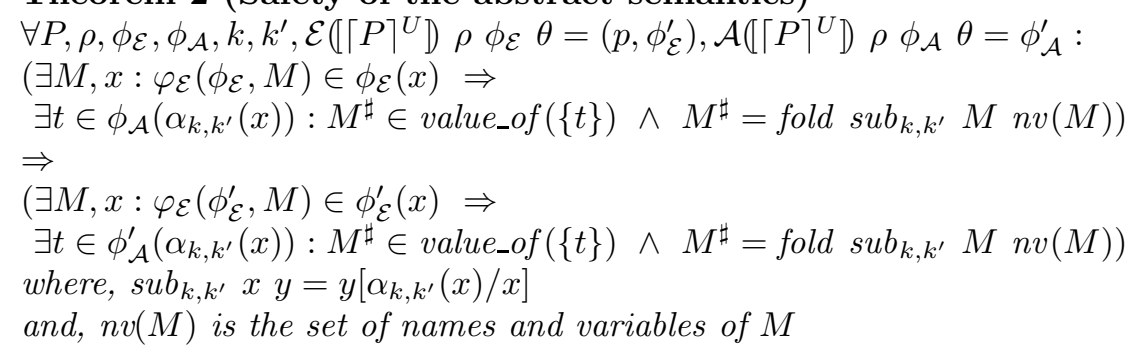

Proof Sketch. The proof is by induction over the structure of the abstract semantics and relies on first proving the safety of the $\cup_{\phi}$ operation.

The theorem states that for any term, $M$, captured in the non-standard semantics by including its $\varphi_{\mathcal{E}}\left(\phi_{\mathcal{E}}^{\prime}, M\right)$ value in the value of a variable, $\phi_{\mathcal{E}}^{\prime}(x)$, then that corresponds to capturing a tag, $t$, in the abstract semantics, by $\phi_{\mathcal{A}}^{\prime}\left(\alpha_{k, k^{\prime}}(x)\right)$. The appropriateness of $t$ is expressed by the ability to obtain (by folding) an abstract form, $M^{\sharp}=$ fold $s_{b} b_{k, k^{\prime}} M n v(M)$, of the concrete term, $M$, by evaluating $t$ using value_of. More concisely, every concrete term, $M$, captured in the non-standard semantics is also captured in the form of the corresponding abstract tag, $t$, in the abstract semantics. From now on, we shall use the following predicate, to denote the property that a term, $M$, is captured by an agent, $B$, sent by another agent, $A$, given the results of an abstract semantics, $\phi_{\mathcal{A}}$, and the constraints, $k$ and $k^{\prime}$ :

$\operatorname{captured}\left(M, A, B, \phi_{\mathcal{A}}, k, k^{\prime}\right) \stackrel{\text { def }}{=}$

$\exists t \in T a g^{\sharp}, x \in \operatorname{dom}\left(\phi_{\mathcal{A}}\right):(t, A, B) \in \phi_{\mathcal{A}}(x) \wedge M^{\sharp} \in$ value_of $(t)$

\section{Security Properties}

In this section, we formalise two security properties that can be checked by the static analysis of the previous section. These properties are the term secrecy and the peer-entity participation. 


\subsection{Term Secrecy}

We formalise the property that a term, $M$, is never leaked to some agent, $U$, with respect to the results of the abstract semantics, $\phi_{\mathcal{A}}$, and using the captured predicate defined in the previous section, as follows.

Property 1 (Secrecy of the term $M$ w.r.t. $U$ )

$\nexists U^{\prime} \in A G: \operatorname{captured}\left(M, U^{\prime}, U, \phi_{\mathcal{A}}, k, k^{\prime}\right)$

From now on, we write the predicate, $\operatorname{secret}(M, U)$, to indicate that $M$ remains secret with respect to $U$.

\subsection{Peer-Entity Participation}

Peer-entity participation means that an agent, $A$, knows to a certain degree of certainty that another agent, $B$, has participated in a session of some protocol in which $A$ is also a participant. In reality, there are many scenarios that this property could be established, both in its one-way and two-way forms. In this section, we discuss one such scenario, where $A$ creates a nonce, $N$, and $N$ is signed by $B$, then, provided that only $B$ has the knowledge of its own private key, $A$ knows that $B$ has just participated in the protocol if it receives back the signature and is able to verify it. The degree of certainty to which $A$ may establish the property depends on whether $A$ performs a certified or an uncertified retrieval of the public key of $B$. In the uncertified case, $A$ may still be able to raise its degree of certainty by relying on trusted third parties to perform the certified public key retrieval.

Based on this, we define the certified peer-entity participation and the uncertified peer-entity participation, as follows.

Property 2 (Certified peer-entity participation of B by $\mathbf{A}$ ) $\operatorname{captured}\left(\theta(B)^{-}, B, B, \phi_{\mathcal{A}}, k, k^{\prime}\right) \wedge$

$\left(\exists t \in T a g^{\sharp}, x \in \operatorname{dom}\left(\phi_{\mathcal{A}}\right): \phi_{\mathcal{A}}(x)=\{(t, A, A)\} \wedge \theta(B)^{+\sharp} \in\right.$ value_of $\left.(t)\right) \wedge$

$\left(\exists U, L:\right.$ captured $\left.\left([\{L\}]_{\theta(B)^{-}}, U, A, \phi_{\mathcal{A}}, k, k^{\prime}\right)\right) \wedge$

$\left(\forall U^{\prime}: U^{\prime} \neq B \Rightarrow \operatorname{secret}\left(\theta(B)^{-}, U^{\prime}\right)\right)$

Property 3 (Uncertified peer-entity participation of $\mathbf{B}$ by $\mathbf{A}$ )

$\operatorname{captured}\left(\theta(B)^{-}, B, B, \phi_{\mathcal{A}}, k, k^{\prime}\right) \wedge$

$\left(\exists t \in T a g^{\sharp}, x \in \operatorname{dom}\left(\phi_{\mathcal{A}}\right):(t, A, A) \in \phi_{\mathcal{A}}(x) \wedge \theta(B)^{+\sharp} \in\right.$ value_of $\left.(t)\right) \wedge$

$\left(\exists U, L:\right.$ captured $\left.\left([\{L\}]_{\theta(B)^{-}}, U, A, \phi_{\mathcal{A}}, k, k^{\prime}\right)\right) \wedge$

$\left(\forall U^{\prime}: U^{\prime} \neq B \Rightarrow \operatorname{secret}\left(\theta(B)^{-}, U^{\prime}\right)\right)$

\section{$7 \quad$ Example}

We consider here an example of mobile protocol authentication taken from [7]. In this example, we have that agent $A$ is running on a small device with computational power insufficient for performing the full certification of the public key of $B$, therefore it relies on a trusted server with much more computational power to complete the verification process and forward its signed nonce to $B$ : 


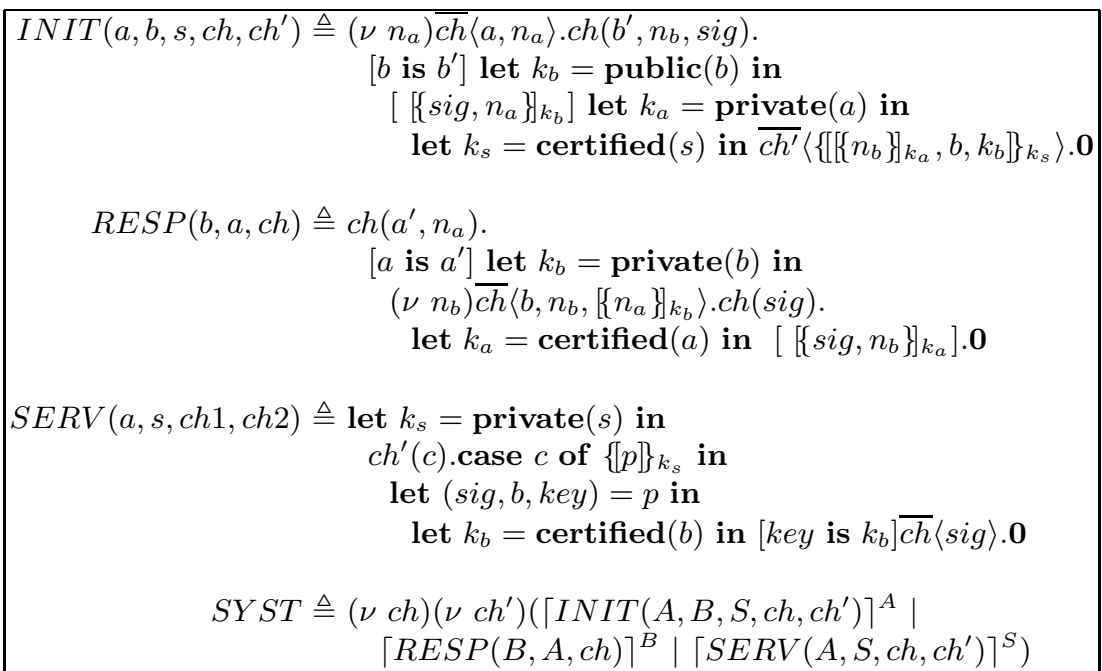

Fig. 6. SPIKY definition of the mobile authentication protocol [7]

$$
\begin{array}{ll}
\text { (1.) } & A \rightarrow B: A, N_{A} \\
\text { (2.) } & B \rightarrow A: B, N_{B},\left[\left\{N_{A}\right\}\right]_{K_{B}} \\
\text { (3.) } & A \rightarrow S:\left\{\left[\left[\left\{N_{B}\right\}\right]_{K_{A}}, B, K_{B}{ }^{+}\right]\right\}_{K_{S}}+ \\
\text { (4.) } & S \rightarrow B:\left[\left\{N_{B}\right\}\right]_{K_{A}}
\end{array}
$$

The specification of this protocol in the SPIKY language is given in Figure 6.

\subsection{Analysis Results}

Applying $\mathcal{A}([S Y S T]) \rho \phi_{\mathcal{A}} \theta$, for the uniform case, where $k=k^{\prime}=1$, we find that Property 3 is satisfied for agent $A$, since captured $\left(\left[\left\{n_{a}\right\}\right]_{k_{b}}, B, A, \phi_{\mathcal{A}}, k, k^{\prime}\right)$ and $A$ can only verify the signature with uncertified public key for $B$. Obviously, $A$ later relies on the server, $S$, to compare the uncertified key of $B$ with a certified version that $S$ is capable of retrieving. On the other hand, we find that Property 2 is satisfied for agent $B$, since captured $\left(\left[\left\{n_{b}\right\}\right]_{k_{a}}, S, B, \phi_{\mathcal{A}}, k, k^{\prime}\right)$ is true and $B$ can verify the signature with a certified public key of $A$. Finally, we also note that Property 1 is satisfied for the term, $\left.\left\{\left[\left\{n_{b}\right\}\right]_{k_{a}}, B, k_{b}\right]\right\}_{k_{s}}$ with respect to all the agents in the second protocol except the server, $\operatorname{SERV}\left(A, S, c h, c h^{\prime}\right)$.

\section{Conclusion}

In this paper, we have presented a novel static analysis of PKI-based systems that captures the property of term substitutions in SPIKY-based specifications. The analysis was used to formally define the security properties of term secrecy and peer-entity participation. For the future, we hope to implement the analysis in some functional language, like SML or OCAML, and we plan then to use the implementation to verify a number of protocols that use PKIs. 


\section{References}

1. Martín Abadi and Andrew Gordon. A calculus for cryptographic protocols: The spi calculus. In Proceedings of the $4^{\text {th }}$ ACM Conference on Computer and Communications Security, pages 36-47, Zurich, Switzerland, April 1997. ACM Press.

2. Samson Abramsky. A domain equation for bisimulation. Information and Computation, 92(2):161-218, June 1991.

3. B. Aziz. A Static Analysis Framework for Security Properties in Mobile and Cryptographic Systems. PhD thesis, School of Computing, Dublin City University, Dublin, Ireland, 2003.

4. B. Aziz, G.W. Hamilton, and D. Gray. A denotational approach to the static analysis of cryptographic processes. In Proceedings of International Workshop on Software Verification and Validation, volume 118, pages 19-36, Mumbai, India, December 2003. Electronic Notes in Theoretical Computer Science.

5. Benjamin Aziz and Geoff Hamilton. A privacy analysis for the $\pi$-calculus: The denotational approach. In Proceedings of the $2^{\text {nd }}$ Workshop on the Specification, Analysis and Validation for Emerging Technologies, number 94 in Datalogiske Skrifter, Copenhagen, Denmark, July 2002. Roskilde University.

6. Benjamin Aziz, Geoff Hamilton, and David Gray. A static analysis of cryptographic processes: The denotational approach. Journal of Logic and Algebraic Programming, 64(2):285-320, August 2005.

7. David Gray, Benjamin Aziz, and Geoff Hamilton. Spiky: A nominal calculus for modelling protocols that use pkis. In Proceedings of the International Workshop on Security Analysis of Systems: Formalism and Tools, Orléans, France, June 2004.

8. Gordon Plotkin. A powerdomain construction. SIAM Journal on Computing, 5(3):452-487, September 1976. 